\title{
69
}

\section{A Distributed Hierarchical Management Framework for Heterogeneous WANs}

\author{
Mark STOVER, Sujata BANERJEE \\ University of Pittsburgh, U.S.A.
}

The scope of network management is expanding in multiple dimensions. Local area networks (LANs) have more nodes than ever before, enterprise networks span national boundaries, wide area networks (WANs) cover the globe, and administrators want to manage their LANs all the way down to a PC's network interface and application software. In order to centrally manage these networks, the network manager faces the complexity of heterogeneous management tools and the difficulty of managing vast amount of data generated by network elements.

Many researchers have begun taking a data-centric view of network management and generally agree that a well-structured global network database is essential for effective network management. One promising paradigm identified by several researchers is to monitor and manage the network through the network management database; however, a number of issues remain-most importantly, the architecture and the data-distribution scheme of the management database. Other important issues include maintaining database consistency, minimizing network management traffic, and interoperation of multiple management standards.

Just as in the research of [1-3], we recognize the importance of a global network management database. Although the MANDATE [1] project proposed a database design that includes the distribution of some data, the focus on a central repository for structural and control data, and the lack of provision for heterogeneous interoperation of multiple management standards, presents a number of difficulties. Our research proposes a fully distributed database with the addition of a new scheme for the hierarchical distribution of network management data.

Our research is driven by these goals: to minimize the network overhead of management data, to create a flexible and scalable management framework that supports multiple management standards, and to provide continued management during network partitioning. The most important aspect of our design is the hierarchical distribution of network management data with multiple management levels. Our design relies upon a distributed database management system (DDBMS) to distribute and replicate the management data. 